\title{
PATTERN FORMATION IN A THIN LAYER OF SEDIMENT
}

\author{
Paolo Blondeaux, Giovanna Vittori and Marco Mazzuoli \\ Department of Civil, Chemical, Environmental Engineering - \\ University of Genova \\ Via Montallegro 1, 16145 Genova, Italy
}

${ }^{1}$ corresponding author, e-mail address: paolo.blondeaux@unige.it, phone: $+39.0103532475$

\section{kewords: coastal bedforms, oscillatory flow, sandy patterns on a rigid substratum}

\begin{abstract}
A stability analysis is performed to investigate the process which leads to the formation of bottom forms, when a thin layer of sediment covers a rigid substratum subject to the oscillatory flow induced by a surface gravity wave. The amplitude of the bottom perturbation is assumed to be so small to linearize the hydrodynamic problem and the flow field is determined by analytical means. However, nonlinear effects are significant and play a fundamental role into the morphodynamic problem, when the rigid substratum is bared by the growth of the most unstable mode. Hence, the development of the bottom profile is evaluated by a numerical approach. The model results show that the wavelength of the morphological patterns, which are generated when the rigid substratum is bared, is longer than the wavelength of the bottom forms which appear when the rigid substratum is always covered
\end{abstract}


1

\section{${ }_{3} 1$ Introduction}

4 Field surveys, carried out along the central part of the strait of Calais-Dover,

5 close to the southern end of the South Fall sand bank, show that the sea

$\sigma$ bed is composed of an almost flat, relic pavement made up of pebbles, sur-

7 mounted by scarce but active sand bedforms (Le Bot \& Trentesaux, 2004).

8

In particular, the field observations show the presence of sand waves which are characterized by wavelengths that appear longer than those of the sand waves generated by tidal currents under the same conditions when the sand is abundant.

Even though the characteristics of the bedforms generated by tidal currents are usually determined by means of linear stability analyses of the flat bottom subject to oscillatory currents, these analyses can not be applied to investigate the process which leads to the appearance of the bottom forms observed by Le Bot \& Trentesaux (2004). In fact, the analyses aimed at investigating the appearance of periodic morphological patterns, like sand waves, are usually carried out by assuming that the bedforms are originated by the growth of the fastest growing mode of a random perturbation of the interface between the flowing water and the sediments. The perturbation is often assumed to be of small amplitude and a linear approach is employed. However, such analyses are carried out by considering the water flowing over a layer of sediment of large (strictly infinite) thickness. 
When, a cohensionless material is superimposed to a "rigid" substratum, the growth of the perturbation profile bares the rigid substratum, even at the early stages of bedform formation, and a linear approach can not be used any longer, even if the amplitude of the bottom waviness is quite small. Indeed, the bottom profile is no longer described by the fastest growing mode and a full nonlinear approach should be used to investigate the interaction of the different spatial components of the bottom profile.

To provide information on the process which leads to the appearance of bottom forms, when only a limited amount of sand is available, we analyse the formation of ripples on a thin layer of sediment lying over a rigid substratum. We choose to investigate the behaviour of ripples instead of the dynamics of sand waves for two main reasons.

First, the mechanism which originates sand waves in tidal environments is similar to that which originates ripples under sea waves. In fact, the interaction of a perturbation of the cohesionless bottom with an oscillatory flow (generated by either wind waves or tidal waves) gives rise to a steady streaming which consists of recirculating cells, the form, intensity and direction of which depend on the hydrodynamic parameters and the characteristics of the bottom undulation. Since the sediment is dragged by the fluid, if the steady velocity component close to the bottom is directed from the troughs towards the crests of the bottom undulation and is strong enough to overcome the effects of gravity, which tends to move the sediment from the crests towards the troughs, the undulation grows and originates the bottom forms. We choose to consider ripple formation instead of sand wave formation, because the investigation of the smaller bedforms allows to solve the hydrodynamic 
problem without the need to introduce uncertainty elements which can mask possible deficiencies of the model, such as a turbulence model and/or the shallow water approximation. The interested reader can look at the books of Allen (1984), Sleath (1984) and Nielsen (1992) and the paper of Sleath, 1976 and those of the group of the University of Genoa (Blondeaux, 1990; Vittori \& Blondeaux, 1990, 1992, 1994, 1996; Foti \& Blondeaux, 1995; Blondeaux \& Vittori, 1996, 1999; Blondeaux et al., 2000; Mazzuoli et al., 2011; Roos \& Blondeaux, 2001).

Secondly, it is easy to make laboratory experiments on the dynamics of ripples over a rigid substratum and to obtain data which can be used to validate the theoretical results.

Needless to write that, even though the appearance of both ripples and sand waves is induced by the steady streaming which is generated by the interaction of an oscillatory flow with a bottom waviness, significant differences exist between the process which leads to the appearance of ripples and that which causes the appearance of sand waves. Some of the differences are implicit in what previously written (for example ripples appear also when the flow regime in the oscillatory boundary layer is laminar while the tidal currents are certainly turbulent). Moreover, the formation of the sand waves is also affected by wind and storms (Le Bot \& Trentesaux, 2004), by biota activity (Borsje et al., 2009) and other phenomena. Nevertheless we think that the results of the present investigation can provide useful information.

In the next section we describe the theoretical analysis. Herein, we give only a brief description of the procedure used to solve the hydrodynamic problem since the procedure follows closely that used by Blondeaux (1990). 
We focus our attention on the evaluation of the sediment transport and the time development of the bottom profile. Section 3 is devoted to describe the results of a few experiments which were carried out to verify that the theoretical approach provides a reliable description of the phenomenon. The last section is devoted to the conclusions and the discussion of the future developments of the work.

\section{The theoretical analysis}

\subsection{Formulation of the problem}

Let us consider a two-dimensional surface gravity wave of small amplitude in a water body of depth $h^{*}$. A thin layer of sediments of uniform size $d^{*}$, density $\rho_{s}^{*}$ and porosity $n$ covers the rigid substratum. Let us denote with $\Delta^{*}$ the thickness of the sediment layer (presently a star denotes a dimensional quantity while the same symbol without a star denotes its dimensionless counterpart).

It is well established that the flow generated by the wave can be modelled as irrotational except within viscous boundary layers adjacent to the bottom and the free surface. Since, we are interested in the interaction between the fluid and the sediment, let us focus our attention on the bottom boundary layer and use the linear wave theory to describe the flow outside this region. We assume the characteristic thickness of the viscous bottom boundary layer $\delta^{*}$ to be much smaller than both the water depth and the length of the gravity wave. Then, the flow in the bottom boundary layer can be considered as caused by fluid oscillations which have an amplitude which does not depend 
on the longitudinal coordinate $x^{*}$, if a progressive wave is considered, or it is parametrically dependent on $x^{*}$, if a standing wave is considered (we define a Cartesian orthogonal coordinate system $\left(x^{*}, y^{*}\right)$ with the $x^{*}$-axis lying on the bottom and parallel to the direction of fluid oscillations, the $y^{*}$-axis being directed upward). If a region, the extent of which is $\mathrm{O}\left(\delta^{*}\right)$, is considered, the flow in the bottom boundary layer can be assumed to be forced by an oscillating pressure gradient described by

$$
\frac{\partial p^{*}}{\partial x^{*}}=\rho^{*} \omega^{*} U_{0}^{*} \sin \left(\omega^{*} t^{*}\right)
$$

where $\rho^{*}$ denotes the constant density of the fluid, $\omega^{*}=2 \pi / T^{*}$ is the angular frequency of the surface wave and $U_{0}^{*}$ is the local amplitude of the velocity oscillations of the fluid close to the bottom but outside the viscous boundary layer.

If the bottom is flat and $d^{*}$ is much smaller than the characteristic thickness $\delta^{*}$ of the bottom boundary layer, the fluid motion is described by the Stokes' solution (1851) and the sediment moves to and fro. Of course, the Reynolds number of the boundary layer is assumed to be smaller than the value for which turbulence appears and the flow regime to be laminar (Blondeaux \& Seminara, 1979; Blondeaux, 1987; Verzicco \& Vittori, 1996; Vittori \& Verzicco, 1998; Costamagna et al., 2003). Hence, $\delta^{*}$ is equal to $\sqrt{2 \nu^{*} / \omega^{*}}$, $\nu^{*}$ being the kinematic viscosity of the water.

Let us consider a bottom profile which can be written in the form

$$
y^{*}=\epsilon^{*} \eta\left(x^{*}, t^{*}\right)=\epsilon^{*} \int_{0}^{\infty}\left[C\left(t^{*}, \alpha^{*}\right) e^{i \alpha^{*} x^{*}}+\text { c.c. }\right] d \alpha^{*}
$$


where $\epsilon^{*}$ is assumed to be much 'smaller' than the boundary layer thickness and the function $\eta$ is written as a Fourier integral, i.e. as the superimposition of different spatial components.

The study of the two-dimensional flow and bottom time development is posed by the vorticity equation, the flow and sediment continuity equations and boundary conditions which force the matching of the flow with the irrotational flow outside the bottom boundary layer and the no-slip condition at the bottom profile.

The evaluation of the flow, when the bottom is wavy, is simplified if a reference frame $\left(\tilde{x}^{*}, \tilde{y}^{*}\right)$ moving with the fluid far from the bottom is introduced, such that

$$
\tilde{x}^{*}=x^{*}+\frac{U_{0}^{*}}{2} \int_{0}^{t^{*}}\left(e^{i \omega^{*} t^{*}}+\text { c.c. }\right) d t^{*}, \quad \tilde{y}^{*}=y^{*}
$$

The transformation (3) reduces the hydrodynamic problem to the determination of the flow generated by the oscillations of a wavy wall in a fluid otherwise at rest.

At this stage, it is convenient to introduce the following dimensionless variables

$$
t=\omega^{*} t^{*}, \quad(x, y, \tilde{x}, \tilde{y})=\frac{\left(x^{*}, y^{*}, \tilde{x}^{*}, \tilde{y}^{*}\right)}{\delta^{*}}, \quad(u, v, \tilde{u}, \tilde{v})=\frac{\left(u^{*}, v^{*}, \tilde{u}^{*}, \tilde{v}^{*}\right)}{U_{0}^{*}}
$$

where $t^{*}$ is time and $\left(u^{*}, v^{*}\right),\left(\tilde{u}^{*}, \tilde{v}^{*}\right)$ are the velocity components along the $\left(x^{*}, y^{*}\right)$ - and $\left(\tilde{x}^{*}, \tilde{y}^{*}\right)$-axes, respectively.

If the stream functions $\psi$ and $\tilde{\psi}$ are introduced such that $\tilde{\psi}=\psi+$ $\frac{1}{2}\left(e^{i t}+\right.$ c.c. $) \tilde{y}$, the functions $\psi$ and $\tilde{\psi}$ for a flat bottom can be easily de- 
termined and turn out

$$
\tilde{\psi}_{0}=\left[C_{1}-\frac{1}{2(1+i)} e^{-(1+i) \tilde{y}}\right] e^{i t}+c . c ., \quad \psi_{0}=\left[C_{2}-\frac{1}{2(1+i)} e^{-(1+i) y}-\frac{1}{2} y\right] e^{i t}+c . c .
$$

2 the stream functions being related to the velocity components by

$$
(u, v)=\left(\frac{\partial \psi}{\partial y},-\frac{\partial \psi}{\partial x}\right), \quad(\tilde{u}, \tilde{v})=\left(\frac{\partial \tilde{\psi}}{\partial \tilde{y}},-\frac{\partial \tilde{\psi}}{\partial \tilde{x}}\right)
$$

3 In (5), $C_{1}, C_{2}$ are arbitrary constants.

4 In the oscillating reference frame, the dimensionless bottom profile turns 5 out to be described by

$$
\tilde{y}=\epsilon \eta\left(\tilde{x}-\frac{R_{\delta}}{4} \int_{0}^{t}\left(e^{i t}+\text { c.c. }\right) d t, t\right)
$$

6 where $\epsilon<<1$ and the Reynolds number is defined by

$$
R_{\delta}=\frac{U_{0}^{*} \delta^{*}}{\nu^{*}}
$$

7 Taking into account that the dimensionless amplitude $\epsilon=\epsilon^{*} / \delta^{*}$ of the bottom 8 waviness is assumed to be much smaller than 1, it is possible to write the 9 stream function $\tilde{\psi}$ in the form

$$
\tilde{\psi}=\tilde{\psi}_{0}+\epsilon \int\left[C(t, \alpha) \tilde{\psi}_{1}(\alpha, \tilde{y}, \tilde{t}) e^{i \alpha \tilde{x}}+c . c .\right] d \alpha
$$

10 Then, by cross-differentiating the momentum equations along the $\tilde{x}$ - and 
$1 \tilde{y}$-axes, vorticity equation is obtained

$$
\frac{\partial}{\partial t}\left(\nabla^{2} \tilde{\psi}\right)+\frac{R_{\delta}}{2}\left[\frac{\partial \tilde{\psi}}{\partial \tilde{y}} \frac{\partial}{\partial x}\left(\nabla^{2} \tilde{\psi}\right)-\frac{\partial \tilde{\psi}}{\partial \tilde{x}} \frac{\partial}{\partial y}\left(\nabla^{2} \tilde{\psi}\right)\right]=\frac{1}{2} \nabla^{2}\left(\nabla^{2} \tilde{\psi}\right)
$$

2 At $O(\epsilon)$, the problem for the generic spatial harmonic component is posed 3 by

$$
\frac{\partial}{\partial t}\left(N^{2} \tilde{\psi}_{1}\right)+\frac{i \alpha R_{\delta}}{2}\left[\frac{\partial \tilde{\psi}_{0}}{\partial \tilde{y}} N^{2} \tilde{\psi}_{1}-\frac{\partial^{3} \tilde{\psi}_{0}}{\partial \tilde{y}^{3}} \tilde{\psi}_{1}\right]=\frac{1}{2} N^{2}\left(N^{2} \tilde{\psi}_{1}\right)
$$

4 where $N^{2}=\left(\partial^{2} / \partial \tilde{y}^{2}\right)-\alpha^{2}$. The no-slip condition and the matching of the 5 solution with the vanishing velocity far from the wall close the problem. 6 Blondeaux (1990) wrote the solution of the (11) in the form

$$
\tilde{\psi}_{1}(\tilde{y}, \tilde{t}, \alpha)=\sum_{m=-\infty}^{\infty} G_{m}(\tilde{y}, \alpha) e^{i m t}
$$

7 where the functions $G_{m}$ are provided by

$$
G_{m}(\tilde{y}, \alpha)=\sum_{n=-\infty}^{\infty}\left\{a_{n} \sum_{j=0}^{\infty} \lambda_{n, m, j} \exp [-[\alpha+(m-n) i+j] \tilde{y}]\right.
$$

8

$$
\left.+\left(1-\delta_{n 0}\right) b_{n} \sum_{j=0}^{\infty} \vartheta_{n, m, j} \exp \left[-\left[\sigma_{n}+(m-n) i+j\right] \tilde{y}\right]\right\}
$$

$$
+b_{0} \sum_{j=0}^{\infty}\left\{\beta_{m, j} \exp [-[\alpha+m i+j] \tilde{y}]+\gamma_{m, j} \tilde{y} \exp [-[\alpha+m i+j] \tilde{y}]\right\}
$$

10 with $\sigma_{n}=\sqrt{\alpha^{2}+2 n i}$. The values of $\lambda_{n, m, j}, \vartheta_{n, m, j}, \beta_{m, j}, \gamma_{m, j}$ are easily found

11 by plugging (13) into (11). For example, the values of $\lambda_{n, m, j}$ are provided by 
1

the following recursive relationship

$$
\lambda_{n, m, j}=\frac{1}{2} i \alpha R_{\delta}\left\{\lambda_{n, m-1, j-1}\left[(\alpha+(m-1-n) i+j-1)^{2}-\alpha^{2}-2 i\right]\right.
$$

2

$$
\left.+\lambda_{n, m+1, j-1}\left[(\alpha+(m+1-n) i+j-1)^{2}-\alpha^{2}-2 i\right]\right\}
$$

$$
\times\left\{\left[(\alpha+(m-n) i+j)^{2}-\alpha^{2}\right]\left[(\alpha+(m-n) i+j)^{2}-\alpha^{2}-2 i m\right]\right\}^{-1}
$$

4

where $\lambda_{m, m, 0}$ can be fixed equal to 1 without loss of generality because of the presence of the constants $a_{n}, b_{n}$. Similar relationships are found for $\vartheta_{n, m, j}, \beta_{m, j}, \gamma_{m, j}$. Finally, $a_{n}$ and $b_{n}$ are determined by forcing the boundary conditions at the bottom. This procedure leads to a system of linear algebaric equations for $a_{n}, b_{n}$, which can be easily solved. Finally, the stream function over the given bottom profile can be computed by making the integral (9).

The determination of the flow field does not differ substantially from that described in Blondeaux (1990).

Once the flow is obtained, the time development of the bottom waviness can be evaluated by considering Exner equation together with a predictive formula to evaluate the sediment transport rate.

In coastal regions, the flow induced close to the bottom by tides and waves often falls in the turbulent regime. Hence, most of the formulae used to predict the sediment transport rate are based on data obtained for values of the parameters such that turbulence effects are predominant and only a few formulae can be found in the literature which can be used when the flow regime is laminar. Since, the theoretical analysis is developed considering a laminar 
1

flow and the experimental data described in the following are characterized by values of the Reynolds number falling in the laminar regime, we use the modified Grass 83 Ayoub's (1982) formula which appears to contain the main physical ingredients controlling the process of sediment transport and can be used when the flow regime is laminar. Moreover, the use of Grass 83 Ayoub's (1982) formula allows an easy comparison of the present results with those of Blondeaux (1990) and Vittori $\&$ Blondeaux (1990), who investigated the formation of ripples on a layer of sediment of infinite thickness.

It follows that the sediment transport rate is predicted by means of

$$
q=1.23 \frac{(s-1)}{s} \frac{\psi_{d}^{2.135}}{R_{p}^{2.45}}\left|2 U-\beta \frac{R_{p}}{\sqrt{\psi_{d}}} \frac{\partial \eta}{\partial x}\right|^{b} \operatorname{sgn}\left(2 U-\beta \frac{R_{p}}{\sqrt{\psi_{d}}} \frac{\partial \eta}{\partial x}\right)
$$

where the dimensionless sediment transport rate per unit width $q$ is given by its dimensional value divided by $\sqrt{(s-1) g^{*} d^{* 3}}$. In (14), $U$ is the dimensionless fluid velocity component parallel to the bottom profile evaluated at a distance equal to $d / 2$ from it, $\beta$ is a friction coefficient and

$$
b=4.28, \quad s=\frac{\rho_{s}^{*}}{\rho^{*}}
$$

Moreover, in (14), $R_{p}$ is the particle Reynolds number and $\psi_{d}$ is the sediment mobility number defined by

$$
R_{p}=\frac{\sqrt{(s-1) g^{*} d^{* 3}}}{\nu^{*}}, \quad \psi_{d}=\frac{U_{0}^{* 2}}{(s-1) g^{*} d^{*}} .
$$

where $g^{*}$ is gravity acceleration. The reader should notice that the Reynolds number $R_{d}$ and the sediment Froude number $F_{d}$ employed by Blondeaux 
${ }_{1}$ (1990) are related to $R_{p}$ and $\psi_{d}$ by

$$
R_{d}=\frac{U_{0} d}{\nu}=R_{p} \sqrt{\psi_{d}}, \quad F_{d}=\sqrt{\psi_{d}}
$$

2

Since the parameter $\epsilon$ is assumed to be small, both the dimensionless velocity $U$ and the sediment transport rate $q$ are expanded as power series of $\epsilon$ and terms of order $\epsilon^{2}$ are neglected.

However, relationship (14) can be applied only when an unlimited supply of sand is available. Therefore, it can be used only when a layer of sediment of large (strictly infinite) thickness is present. If, locally, no sand is present and the bottom turns out to be rigid, the value of the sediment transport rate depends not only on the local value of $U(x, t)$ but also on its spatial derivative $\partial U(x, t) / \partial x$, as discussed below.

Let us consider a phase of the oscillating cycle such that $U(x, t)$ is positive. If, at the generic coordinate $x$, no sand is available and $\partial U(x, t) / \partial x$ is positive, the sediment transport rate can not increase because no further sediment can be mobilized. Hence, $\partial q / \partial x$ should be forced to vanish and the sediment transport rate at $x$ equals to the sediment trasport rate at $x-d x$. On the other hand, if $\partial U(x) / \partial x$ is negative, the value of the sediment transport rate at $x$ depends on the value of $q$ at $x-d x$. Indeed, if the value of $q$ at $x-d x$ is larger than that provided by (14) evaluated at $x$, the local sediment transport rate at $x$ can be determined by means of (14) and some sand is deposited at the bottom according to sediment continuity equation. On the other hand, if the value of $q$ at $x-d x$ is smaller than that provided by (14) at $x$, the local sediment transport rate at $x$ should be assumed to be 
7 where

$$
q=Q \widehat{q}, \quad \tau=t Q \frac{R_{p}}{2(1-n)}, \quad Q=1.23 \frac{(s-1) \psi^{2.135}}{s R_{p}^{2.45}}\left(\frac{R_{p} \sqrt{\psi_{d}}}{R_{\delta}}\right)^{b}
$$

8 in such a way that $\widehat{q}$ is a function of order one and $\tau$ is the morphodynamic 9 temporal variable.

equal to that at $x-d x$. Similar relationships hold when $U(x, t)$ is negative.

Once the sediment transport rate is evaluated as a function of $x$, following the simple rules previously described, the time development of the bottom profile can be determined by the integration of sediment continuity equation (Exner equation), which in dimensionless form reads

$$
\frac{\partial \eta}{\partial t}=-\frac{R_{p}}{2(1-n)} \frac{\partial q}{\partial x}
$$

$$
\frac{\partial \eta}{\partial \tau}=-\frac{\partial \widehat{q}}{\partial x}
$$

\subsection{Numerical approach}

As already pointed out, formula (14), which quantifies the sediment transport rate when the bottom is an "infinite" source of sediment, can be linearized taking into account that the value of $U$ at $y=d / 2$ is equal to $\partial \psi_{0} / \partial y$ at $y=d / 2$ plus a small correction of $O(\epsilon)$. However, the simple rules, which are introduced to evaluate the sediment transport rate when the rigid substratum is bared, introduce strong nonlinearities and the bottom time development 
should be obtained by numerical means. A finite difference approach is used and the time advancement of (18) is obtained by means of a Runge-Kutta approach of second order while the spatial derivatives are replaced by their finite difference (second order) approximation.

Equation (18) is integrated starting from a small random initial pertubation and the integration is carried out till a "regime" configuration is attained. A computational domain of length $L_{b o x}=L_{b o x}^{*} / \delta^{*}$ along the $x$-axis $\left(x=x^{*} / \delta^{*}\right)$ is considered and periodic boundary conditions are applied at $x=0$ and $x=L_{b o x}$. Needless to write that the value of $L_{b o x}$ is chosen large enough to assume its influence on the time development of the bottom configuration to be negligible. Denoting with $\Delta x$ and $\Delta t$ the grid size and the time step used in the numerical integration of (19), some of the numerical simulations were repeated halving both the grid size and the time step. The results indicate the chosen value of $\Delta x$ and $\Delta t$ do not affect the time development of the bottom.

\section{$2.3 \quad$ Numerical results}

An exhaustive investigation of the phenomenon in the parameter space is not possible because of the large number of the parameters which affect the time development of the bottom configuration. Moreover, a detailed analysis of the results is not within the aims of the present work which is simply devoted to test whether the approach summarized in sections 2.1 and 2.2 can provide a reliable description of the formation of bottom forms when the rigid substratum is bared.

Figure 1 shows the bottom profile at the beginning of the first numerical 
simulation and at the end of the simulation, when the bottom profile attains a quasi-equilibrium configuration. The values of the flow and sediment parameters are: $\beta=0.15, R_{\delta}=33.87, s=1.27, R_{p}=2.99$ and $\psi_{d}=12.96$. These values of the parameters are chosen because they fall in the range of the values typical of the experiments described in the following section. In particular, these values correspond to naylon pellets $\left(\rho_{s}^{*}=1270 \mathrm{~kg} \mathrm{~m}^{-3}\right)$, characterized by a mean grain size $d^{*}$ equal to $0.15 \mathrm{~mm}$ and dragged by the flow at the bottom of a node of a standing wave which is characterized by a period $T^{*}$ equal to $0.7 \mathrm{~s}$, a height $H^{*}$ equal to $2 \mathrm{~cm}$ and evolves on a constant water depth $h^{*}$ equal to $10 \mathrm{~cm}$ (the length $L^{*}$ of the wave turns out to be $60 \mathrm{~cm}$ ). The dimensionless length of the numerical box is equal to 100 and the dimensionless volume of the sand which covers the bottom is equal to 7.5 which corresponds to a dimensionless thickness of the sediment layer equal to 0.075 . Figure 1 shows that, at the beginning of the simulation, the bottom is flat but for a random perturbation of small amplitude. At the end of the simulation, 14 ripples are present, which are separated by regions of bared rigid substratum. The reader should take into account that the vertical axis of figure 1 is magnified and the height of the equilibrium ripples is much smaller than their length. In particular, it is always verified that the ratio between the height of the ripples and their length is smaller than 0.1, which is the threshold value suggested by Sleath (1984) below which flow separation can be assumed to be absent. Hence, the flow does not separate at the ripple crests and the linear approach appears appropriate to describe the flow field.

At the beginning of the simulation, the perturbation components characterized by the smallest wavelengths rapidly decay and only the undulations 
characterized by wavelengths of the order of the viscous length $\delta^{*}$ or larger survive. Figure 2 shows the initial bottom profile and the bottom profile at $t=4 \pi$, i.e. after two oscillating cycles, in the region $0 \leq x \leq 10$ to allow a clearer evaluation of the results. This first part of the numerical simulation can be thought more as a numerical trick to obtain a reasonable initial bottom configuration rather than a physically meaningful description of the time development of the bottom profile. Indeed, the initial waviness, which is generated as a sequence of random numbers, is characterized by a so short wavelength and the sediment is so light that the temporal morphodynamic scale is not much larger than the wave period and the changes of the bottom profile, taking place during a wave period, could not be neglected. At later stages, because of the increase of the wavelength of the bottom forms, the time development of the bottom profile is much slower and the assumtions underlying the model are verified.

The results show that the fastest growing mode predicted by the linear stability analysis of Blondeaux (1990) slowly prevails on the other modes and 23 ripples appear (see figure 3 ). The amplitude of the bottom forms grows till their troughs touch the rigid substratum at a few locations (see figure 4). At later stages, the time development of the profile of the bedforms bares the rigid substratum (see figure 5) and, later, different phenomena take place, which are induced by the strong nonlinear effects caused by the presence of the rigid substratum.

For example, some of the bottom forms annihilate and the average spacing between the crests suddenly increases when a bottom form disappears (see figure 6). In other cases, a ripple disappears because two crests migrate each 
towards the other till they coalesce thus giving rise to a single sediment bump and a sudden increase of the average spacing of the ripples (see figure 7).

At later stages, the time development of the bottom profile slows down further and a quasi equilibrium is attained. However, a small transfer of sediments among the different ripples still takes place, some of the bottom forms slowly migrate to make uniform the spacing between the crests and the amplitude of the bottom forms slightly oscillates in time. Moreover, small differences are observed between the height of one ripple and the height of the adjacent ripples. These differences are due to the nonlinear dynamics of the bottom forms which tends to generate finite amplitude variations of the ripple height. Figure 8 shows the average ripple wavelength plotted versus time.

It is worthwhile to point out that the numerical simulation was made for a value of the friction coefficient $\beta$ equal to 0.15 , which is the value used by Blondeaux (1990). Since in the literature no well established value of $\beta$ can be found and Fredsoe (1974) pointed out that $\beta$ should range between 0 and 1 , the simulation was repeated for $\beta=0.25, \beta=0.5$ and $\beta=0.75$. The qualitative behaviour of the time development of the bottom perturbation is not affected by the value of $\beta$, even though larger values of $\beta$ lead to longer ripples. For example, for $\beta=0.15$ the final crest-to-crest distance is about $4.5 \delta^{*}$, while for $\beta=0.25, \beta=0.5$ and $\beta=0.75$, the crest-to-crest distance turns out to be $4.8 \delta^{*}, 12.5 \delta^{*}$ and $16.7 \delta^{*}$, respectively.

By keeping fixed the sediment characteristics $\left(d^{*}=0.15 \mathrm{~mm}, \rho_{s}^{*}=1270\right.$ $\mathrm{kg} \mathrm{m}^{-3}$ ) and considering a standing wave of height and length equal to those of the first numerical simulation $\left(H^{*}=2 \mathrm{~cm}, L^{*}=60 \mathrm{~cm}\right)$, results were 
obtained by varying the water depth $h^{*}$, which implies different amplitudes $U_{0}^{*}$ and periods $T^{*}$ of the fluid velocity oscillations close to the bottom. Ripple formation was analysed under a node of the standing wave and fixing the ratio $\Delta$ between $\Delta^{*}$ and $\delta^{*}$ equal to 0.1 .

Figure 9 shows the time development of the ripple wavelength for two different values of the water depth, namely $h^{*}=10 \mathrm{~cm}$ and $h^{*}=12.5 \mathrm{~cm}$. The wavelength plotted in the figure is the average value of the crest-tocrest distance observed in the computational domain, where the amplitude of the velocity oscillations is independent of the $x$-coordinate, because the surface waves are assumed to be characterized by a length much longer than the ripple size. The increase of the water depth leads to a decrease of the amplitude of the fluid velocity oscillations close to the bed. It follows that the sediment mobility number decreases and the morphodynamic time development of the bottom slows down as shown by (14) and (18). Moreover, figure 9 shows that the dimensionless length of the bottom forms $L_{r}=L_{r}^{*} / \delta^{*}$ at equilibrium decreases from values slightly larger than 7 for $h^{*}=10 \mathrm{~cm}$ (see the top panel of figure 9) to values slightly larger than 6 for $h^{*}=12.5$ $\mathrm{cm}$ (see the bottom panel of figure 9). However, the equilibrium wavelength of the ripples is not a monotonic function of the water depth as shown by figure 10, where the time development of $L_{r}$ is plotted versus $\tau$ for $h^{*}=7.5$ $\mathrm{cm}$ and the equilibrium wavelength turns out to fall between 4 and 5 . The variations of $L_{r}^{*}$ with $h^{*}$ can be qualitatively understood following the lead of Blondeaux (1990). During an oscillating cycle, the sediment particles feel the influence of the steady recirculating cells which form over the ripple. The number of steady recirculating cells, which affect the dynamics of sediment 
particles, suddenly increases when the ratio $\hat{r}$ between the amplitude of the sediment displacement oscillations and the ripple wavelength crosses the values $n / 2$ with $n=1,2, \ldots$. The quantitative prediction of $\hat{r}$ is not simple and the reader can look at figure 6 of Blondeaux (1990) which shows the ripple wavenumber $2 \pi \delta^{*} / L_{r}^{*}$ as function of the Reynolds number $R_{\delta}$ for different sediment sizes, when the layer of sediment particles can be assumed to be of infinite thickness.

Further numerical simulations were made for different values of $H^{*}$ and keeping the other dimensional quantities equal to those of the first numerical simulation. No qualitative change of the results is observed when the value of $H^{*}$ is increased. However, if $H^{*}$ is decreased beyond a critical value $H_{c r i t}^{*}$, the results show that any initial bottom perturbation decays. In other words, the flat bottom configuration appears to be stable when $H^{*}$ is smaller than $H_{c r i t}^{*}$. For example, figure 11 shows the bottom profile for $H^{*}=1 \mathrm{~cm}$ at the beginning of the simulation and at $t=4,40,400$. This numerical finding is in agreement with the linear stability analysis of Blondeaux (1990) which shows that relatively small values of the sediment mobility number lead to the stability of the flat bottom configuration.

At last, a series of numerical simulations was made for values of the dimensional parameters equal to those of the first simulation but varying the thickness of the mobile sediment layer covering the rigid substratum. For example, figure 12 shows the time development of the ripple wavelength for the same values of the parameters as those of figure 8 but for a thicker layer of mobile sediment. The initial time development of the average spacing of the ripples is similar and in both cases the ripple wavelength seems to converge 
towards a value of about 4. However, as time passes, the rigid substratum is bared earlier for $\Delta=0.075$ than for $\Delta=0.15$, even though the final crest-to-crest distance is equal.

The results of all the numerical simulations for different values of $\Delta$ are summarized in figure 13. The model shows that the number of ripples increases as $\Delta$ is increased till an asymptotic value is attained. Hence, it follows that the average crest-to-crest distance ripple is a decreasing function of $\Delta$ but with a horizontal asymptote which is attained for increasing values of $\Delta$. However, let us point out that results for large values of $\Delta^{*}$ were not obtained because the equilibrium amplitude of the ripples increases as $\Delta^{*}$ increases and for large values of the ripple amplitude, the flow separates from the ripple crests, vortices are generated and the linear approach employed to determine the flow field is not appropriate. Similarly, the reader should be aware that the results for very small values of $\Delta$ are meaningless and are not obtained, because the assumption of a continuous distribution of sediment becomes questionable when $\Delta^{*}$ becomes comparable with the grain size $d^{*}$.

Even though the theoretical approach seems to provide a reliable description of the phenomenon under investigation, at least a qualitative comparison of the theoretical results with experimental observations appears to be necessary to validate the theoretical analysis and verify the main findings of the model results: 1) the wavelength of the bottom forms increases when the rigid substratum is bared and 2) the final crest-to-crest distance appears to be independent of the thickness of the layer of mobile sediment, if $\Delta^{*}$ is neither too small nor too large. Indeed, the numerical simulations show that the crest-to-crest distance increases when $\Delta$ tends to vanish. However, 
1 the increase of $L_{r}$ takes place for values of $\Delta$ so small that the thickness $2 \Delta^{*}$ of the mobile sediments is comparable with the sediment size $d^{*}$ and the 3 continuum approach used to describe the time development of the bottom 4 profile becomes questionable (the reader can easily compute the ratio $\Delta^{*} / d^{*}$ 5 by noticing that this ratio is equal to $\left.\Delta R_{\delta} /\left(R_{p} \sqrt{\psi_{d}}\right)\right)$.

\section{${ }_{6} 3$ The laboratory experiments}

\section{3.1 The apparatus and the experimental procedure}

8 The experiments were carried out in a small tank, which was $30 \mathrm{~cm}$ long, $95 \mathrm{~cm}$ large and $30 \mathrm{~cm}$ high. The tank was made of a transparent material 10 (polymethyl methacrylate) that allows the observation of the motion of both 11 the water, which fills the tank, and the sediments. The density $\rho^{*}$ of the

\footnotetext{
water and its kinematic viscosity $\nu^{*}$ can be assumed to be $1000 \mathrm{~kg} / \mathrm{m}^{3}$ and $10^{-6} \mathrm{~m}^{2} / \mathrm{s}$, respectively, since the water temperatude was about $18^{\circ}$ Celsius. The sediments, which were spread over the bottom of the tank, were nylon pellets characterized by a mean grain size $d^{*}$ equal to $0.15 \mathrm{~mm}$ and a density $\rho_{s}^{*}$ equal to $1270 \mathrm{~kg} / \mathrm{m}^{3}$. The tank was mounted over a tray which oscillated in the horizontal direction. The angular frequency $\omega^{*}$ of the oscillations was chosen equal to the frequency of the standing wave inside the tank, which depends on the water depth $h^{*}$ and the length $\ell^{*}$ of the tank
}

$$
\omega^{*}=\sqrt{\frac{g^{*} \pi}{\ell^{*}} \tanh \left(\frac{\pi h^{*}}{\ell^{*}}\right)} .
$$


On the other hand, the amplitude $A^{*}$ of the tank oscillations could be varied to obtain different values of the height $H^{*}$ of the standing wave, which was generated within the tank by its oscillations.

The flow generated inside the tank can be considered irrotational but for the presence of viscous boundary layers adjacent to the bottom and the lateral walls. If the height $H^{*}$ of the standing wave was small compared with $2 \ell^{*}$, close to the bottom but outside the viscous boundary layer, the fluid oscillated harmonically with a velocity described by

$$
u^{*}\left(x^{*}, t^{*}\right)=U_{0}^{*}\left(x^{*}\right) \cos \left(\omega^{*} t^{*}\right)
$$

whe axc tank oscillations) and the $y^{*}$-axis pointing upwards. The amplitude $U_{0}^{*}\left(x^{*}\right)$ of the velocity oscillations can be easily evaluated and turns out to be

$$
U_{0}^{*}\left(x^{*}\right)=\frac{\pi g^{*} H^{*}}{2 \omega^{*} \ell^{*} \cosh \left(\frac{\pi h^{*}}{\ell^{*}}\right)} \sin \left(\frac{\pi x^{*}}{\ell^{*}}\right)
$$

An experiment started when the motor of the tray was switched on, fixing the values of $\omega^{*}$ and $A^{*}$. The motor was switched off after $N_{1}$ cycles and the geometrical characteristics of the ripples were measured. Then, the tank was oscillated again for $N_{2}$ cycles and the ripple wavelength was measured again. This procedure was repetead till the ripples attained an equilibrium configuration. The crest-to-crest distance of the bottom forms was evaluated by measuring the length of the region occupied by $N_{r}$ ripples in the central part of the tank and divinding by $N_{r}$. The number $N_{r}$ of ripples varied 
depending on the form of the bottom profile, even though usually five ripples were considered. Figure 14 shows the ripples observed at the bottom of the tank during one of the experiments. The photo shows that the sediments were piled up in a periodic pattern (ripples) but in the troughs of the bottom forms no sediment could be found and the rigid bottom was bared. Figure 14 shows also that the bottom forms are irregular. Hence, even though the length of the ripples is the average over five values, a significant error affects the measurements. To increase the statistical sample and to provide the order of magnitude of the error affecting the measurements, an experiment was repeated three times. The reader can verify that the size of the region occupied by five ripples is significantly smaller than the length of the standing wave generated inside the tank and the amplitude of the velocity oscillations outside the bottom boundary layer in the region occupied by five ripples can be safely assumed to be independent of the coordinate $x^{*}$.

As already pointed out, the aim of the experiments was simply to verify that the theoretical model provides a reliable description of the phenomenon. Hence, only a relatively small number of experiments was made to verify that the crest-to-crest distance follows the qualitative behaviour predicted by the theoretical model.

In the first series of experiments the water depth was set equal to $h^{*}=10$ $\mathrm{cm}$, which gives rise to a period of the standing wave equal to $T^{*}=0.70$ $\mathrm{s}$, and the thickness $\delta^{*}$ of the viscous boundary layer, which is generated close to the bottom, is equal to $0.47 \mathrm{~mm}$. The amplitude of tray oscillations was chosen such that the height of the standing wave inside the tank was $H^{*}=2.0 \mathrm{~cm}$. The amplitude of the velocity oscillations, close to the bottom 
and under the node of the surface oscillations, i.e. in the central part of the tank, can be obtained by means of (22) with $x^{*}=\ell^{*} / 2$ and turns out to be $5.0 \mathrm{~cm} \mathrm{~s}^{-1}$. The flow Reynolds number $R_{\delta}$, the sediment Reynolds number $R_{p}$ and the mobility number $\psi_{d}=\left(U_{0}^{*}\right)^{2} /\left[(s-1) g^{*} d^{*}\right]$ are equal to $33.87,2.99,12.93$, respectively. The volume $V^{*}$ of sediment was $0.5 \mathrm{~cm}^{3}$. The sediments were poured into the tank and allowed to settle down in still water, after an appropriate mixing which was aimed at generating their uniform distribution. At the beginning of the experiment, the sediment (which was uniformly distributed over the bottom of the tank) moved towards the central part of the tank because of the steady streaming which was generated by the nonlinear dynamics of the flow in the boundary layer at the bottom of the standing wave. No attempt was made to measure the thickness of the layer of sediment in the central part of the tank to avoid any perturbation of the sediment spatial distribution. Then, irregular morphological patterns appeared till, after a dozen of cycles, ripples appeared, the wavelength of which could be measured.

The crest-to-crest distance of the ripples changed during the experiments. Figure 15a shows the average wavelength of the ripples as function of the number of oscillating cycles and summarizes the results of the first three experiments of test n.1 while the ensemble averaged value is plotted in figure 15b. The experimental observations, similarly to the theoretical analysis, show that at the beginning of the experiment, ripples appear, the wavelength of which is that of the fastest growing mode of a random perturbation of the interface between sediment and water, which could be predicted by means of the analysis described in Blondeaux (1990). As the amplitude of the bottom 
perturbation grows, sediment crests are observed which are separated by the rigid bottom and, then, the crest-to-crest distance of the bottom forms increases. Hence, both the numerical simulation and the experimental observations show that the final crest-to-crest distance is larger than the wavelength of the fastest growing mode. In particular, test n.1 shows an increase of the wavelength of about $80 \%$, which is equal to the increase obtained by means of the theoretical approach. However, the equilibrium average wavelength of the morphological patterns observed during the experiments differs quantitatively from that which can be predicted by the theoretical model. This is not an unexpected result since the sediment transport predictors are usually affected by a large uncertainty. Indeed, the comparison between the theoretical results and field/laboratory observations of morphological patterns are usually carried out plotting the data and the model results using logarithmic scales. In other words, a morphological stability analysis is commonly considered to be successful when it is able to predict the qualitative behaviour of the bottom forms and the order of magnitude of their geometrical characteristics (see for example Dood et al., 2003).

Since the theoretical model shows that the crest-to-crest spacing does not depend on the value of $\Delta^{*}$, as long as $\Delta^{*}$ is neither too small nor too large, further experiments were made by fixing $h^{*}=10 \mathrm{~cm}, H^{*}=2 \mathrm{~cm}$ and spreading different volumes of sediment at the bottom, i.e. $1 \mathrm{~cm}^{3}$ (test n.2) and $2 \mathrm{~cm}^{3}$ (test n.3). Figures 16a and 17a show the time development of the ripple wavelength for these two cases. Each figure shows three lines because an experiment was repeated three times. These results, along with those plotted in figure 8 support the theoretical model. Indeed, if the average values 
of the ripple wavelength versus time are plotted for the different values of $V^{*}$ (see figure 18), the final ripple wavelength appears to be almost independent of $V^{*}$. The theoretical model shows also that the crest-to-crest distance tends to increase when $\Delta$ tends to vanish. No attempt was made to verify this trend experimentally since it is quite difficult to control the initial thickness of the sediment layer where the ripples appear. Indeed, as already pointed out, the sediments which are initially uniformly spread on the bottom of the tank, tend to move towards the node of the standing wave and it is not possible to fix a priori the value of $\Delta$ with a sufficient accuracy.

On the other hand, the theoretical model shows that the characteristics of the morphological patterns depend on the other parameters of the problem. This theoretical finding is confirmed by other experiments which were made changing the water depth inside the tank. It follows that also the period $T^{*}$ of the standing wave inside the tank changed along with the amplitude of the velocity oscillations induced by the wave close to the bottom and the thickness $\delta^{*}$ of the viscous bottom boundary layer. These changes of the flow field induced modifications of the ripples. In particular, figures 19 and 20 , where the dimensionless average wavelength of the ripples observed for different water depths, is plotted versus time, show that the equilibrium values of the dimensionless crest-to-crest distance of the bottom forms observed for $h^{*}=7.5 \mathrm{~cm}$ and $12.5 \mathrm{~cm}$ turn out to be about 30 and 20, respectively, and are different from the value observed for $h^{*}=10 \mathrm{~cm}$ which was equal to about 27 (the dimensional wavelengths are about $1.5,0.9$ and $1.3 \mathrm{~cm}$ for $h^{*}=7.5,12.5$ and $10 \mathrm{~cm}$, respectively). 


\section{Conclusions and future developments}

Both the theoretical analysis and the experimental observations show that the bedforms, which are generated by an oscillatory flow over a layer of sediment so thin that the bedform appearance bares the underlying rigid substratum, are longer than those which are generated, for the same hydrodynamic conditions, when the thickness of the sediment layer is so thick that the underlying rigid substratum is always covered by the sediment. Moreover, if the thickness $\Delta^{*}$ of the layer of mobile sediment is not too large, the wavelength of the bottom forms seem to be independent on $\Delta^{*}$. These theoretical findings are supported by a comparison of the model results with laboratory observations made to test the theoretical model using a small experimental apparatus.

The analysis assumes that the bottom forms are of small amplitude such that the hydrodynamic problem can be linearized. However, even though the bottom forms are characterized by a small amplitude, their dynamics turns out to be highly nonlinear when the rigid substratum is bared because of the sediment transport predictor which should take into account that no sediment can be further mobilized when even high shear stresses act on a rigid substratum.

If a simple turbulence model is introduced, the analysis could be applied to investigate the bottom forms generated by tidal currents acting on a thin layer of mobile sediments superimposed to a rigid substratum and to explain the formation of the morphological patterns observed by Le Bot \& Trentesaux (2004) in the Calais-Dover strait, where the crest-to-crest distance of the periodic bedforms observed to be larger in the areas where the mobile 


\section{-Acknowledgements} bottom profile.

\section{References}

sediments are not sufficient to cover the pebble lags. However, to tackle this investigation, also the sediment transport predictor should be modified to take into account not only the presence of a turbulent flow but also that relatively small amounts of sand grains can fill the gaps between the pebbles and, hence, they can be eroded and/or deposited without modifying the

This study has been funded by the Office of Naval Research (U.S.A.) (under the research project n. N6290914PR00165) and by the 'Ministero dell'Istruzione, dell'Universitá e della Ricerca' (under the research project n.2012BYTPR5 'Hydro-morphodynamic modelling of coastal processes for engineering purposes'). The support of the University of Genova under the projects PRA2013 and PRA2014 is also acknowledged. The authors thank also Mr. Franco Porcile who helped them to realise the experimental apparatus and Dr. Eva Blondeaux for the photos of the experiments.

[Allen (1984)] Allen, J.R.L. 1984 Sedimentary structures. Their character and physical basis. Developments in sedimentology. 30, Elsevier.

[Blondeaux (1987)] Blondeaux, P. 1987 Turbulent boundary layer at the bottom of gravity waves. J. Hydraulic Res. 25 (4), 447-464.

[Blondeaux (1990)] Blondeaux, P. 1990 Sand ripples under sea waves: Part I. Ripple formation J. Fluid Mech. 218, 1-17.

[Blondeaux and Seminara (1979)] Blondeaux, P. \& Seminara, G. 1979 Transizione incipiente al fondo di un'onda di gravitá. 67, 408-411, (italian) Acc. Naz. Lincei 67, 408-411 (in italian) 
[Blondeaux and Vittori (1994)] Blondeaux, P. \& Vittori, G. 1994 Wall imperfections as a triggering mechanism for Stokes-layer transition. J. Fluid Mech. 264, 107-135.

[Blondeaux and Vittori (1999)] Blondeaux, P. \& Vittori, G. 1999 Boundary layer and sediment dynamics under sea waves. Adv. Coastal and Ocean Engineering 4, 133-190.

[Blondeaux et al. (2000)] Blondeaux, P., Vittori, G. \& Foti E. 2000 Migrating sea ripples. European J. Mechanics B - Fluids 19, 285-301.

[Borsje et al. (2000)] Borsje, B.W., De Vries, M.B., Bouma, T.J., Besio, G., Hulscher, S.J.M.H. \& Herman, P.M.J. 2009 Modeling bio-geomorphological influences for offshore sandwaves. Cont. Shelf Res. 29 (9), 1289-1301.

[Costamagna et al. (2003)] Costamagna, P., Vittori, G. \& BlonDEAUX, P. 2003 Coherent structures in oscillatory boundary layers. $J$. Fluid Mech. 474, 1-33.

[Dodd et al. (2003)] Dodd, N., Blondeaux, P., Calvete, D., De Swart, H.E., Falques, A., Hulscher, S.J.M.H., Rozynski, G. \& Vittori, G. (2003) Understanding coastal morphodynamics using stability methods. J. Coastal Res. 19 (4) 849865.

[Foti \& Blondeaux (1995)] Foti, E. \& Blondeaux, P. 1995. Sea ripple formation: the turbulent boundary layer case. Coastal Eng. 25 (3-4), 227-236.

[Grass \& Ayoub (1982)] Grass, A.J. \& Ayoub, R.N.M. 1982 Bed load transport of fine sand by laminar and turbulent flow. 8th Coastal Engineering Conference, Cape Town, South Africa A.S.C.E. (ed. B. L. Edge), 1589-1599.

[Le Bot \& Trentesaux (2004)] Le Bot, S. \& Trentesaux, A. 2004 Types of internal structure and external morphology of submarine dunes under the influence of tidal and wind-driven processes (Dover Strait, northern France). Marine Geology 211 (1-2), 139-164.

[Mazzuoli et al. (2011)] Mazzuoli, M., Vittori, G. \& Blondeaux, P. 2011 Turbulent spots in oscillatory boundary layers. J. Fluid Mech. , 685, 365-376. 
[Nielsen (1992)] Nielsen, P. 1992. Coastal Bottom Boundary Layers and Sediment Transport. Adv. Series on Ocean Eng. 4, World Scientific.

[Roos \& Blondeaux (2001)] Roos, P.C. \& Blondeaux, P. 2001. Sand ripples under sea waves. Part 4. Tile ripple formation. J. Fluid Mech. 447, 227-246.

[Sleath (1976)] Sleath, J.F.A. 1976 On rolling-grain ripples. J. Hydraul. Res. 14 (1), 69-81.

[Sleath (1984)] Sleath, J.F.A. 1984 Sea Bed Mechanics Wiley Interscience.

[Stokes (1851)] Stokes, G.G. 1851 On the effect of the internal friction of fluids on the motion of pendulums. Cambridge Philos. Trans. 9, 8-106. Reprinted in Mathematical and Physical Papers, 2nd ed., Vol. 3. New York: Johnson Reprint Corp., p. 1, 1966

[Verzicco \& Vittori (1996)] Verzicco, R. \& VitTori. G. 1996 Direct simulation of transition in Stokes layers. Phys. Fluids 8 (6), 1341-1343.

[Vittori \& Blondeaux (1990)] Vittori, G. \& Blondeaux, P. 1990 Sand ripples under sea waves Part 2. Finite-amplitude development. J. Fluid Mech. 218, 19-39.

[Vittori \& Blondeaux (1992)] Vittori, G. \& Blondeaux, P. 1992 Sand ripples under sea waves. Part 3. Brick-pattern ripple formation. J. Fluid Mech. 239, 23-45.

[Vittori \& Blondeaux (1996)] Vittori, G. \& Blondeaux, P. 1996 Mass transport under sea waves propagating over a rippled bed. $J$. Fluid Mech. 314, 247-265.

[Vittori \& Verzicco (1998)] Vittori, G. \& Verzicco, R. 1998 Direct simulation of transition in an oscillatory boundary layer. J. Fluid Mech. 371, 207-232. 

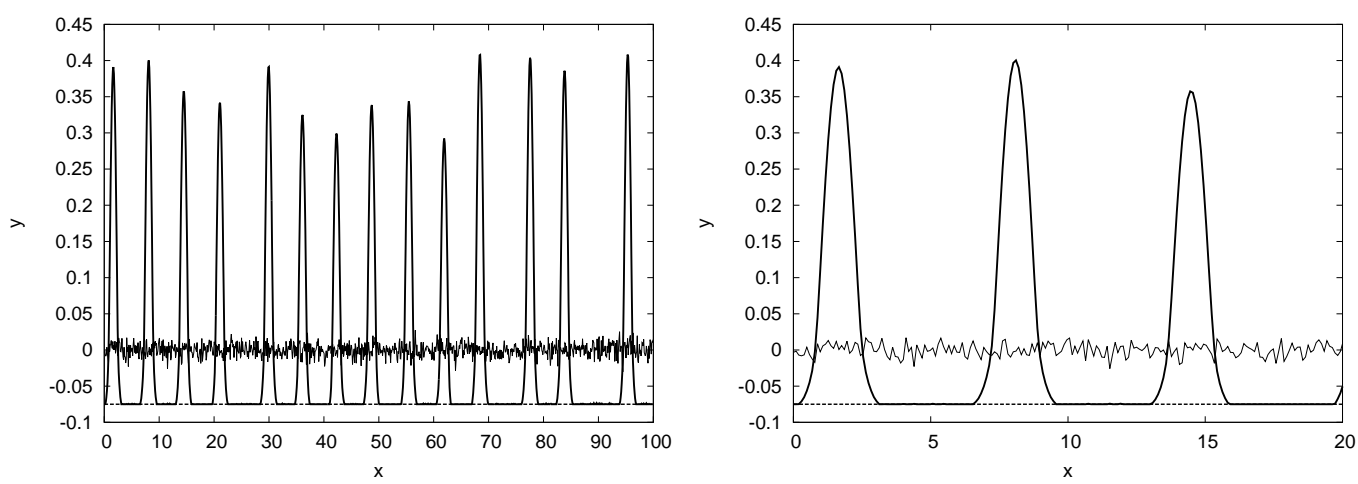

Figure 1: Bottom profile at the beginning of the numerical simulation (thin line) and at the end of the simulation (thick line) when the bottom configuration is quasi-steady. The rigid bottom is located at $y=-0.075$ (thick broken line) $\left(R_{\delta}=33.87, s=1.27, R_{p}=2.99, \psi_{d}=12.96\right)$. The right hand panel is an enlargement of the region $0 \leq x \leq 20$.

\section{${ }_{27} 5 \quad$ Figures}




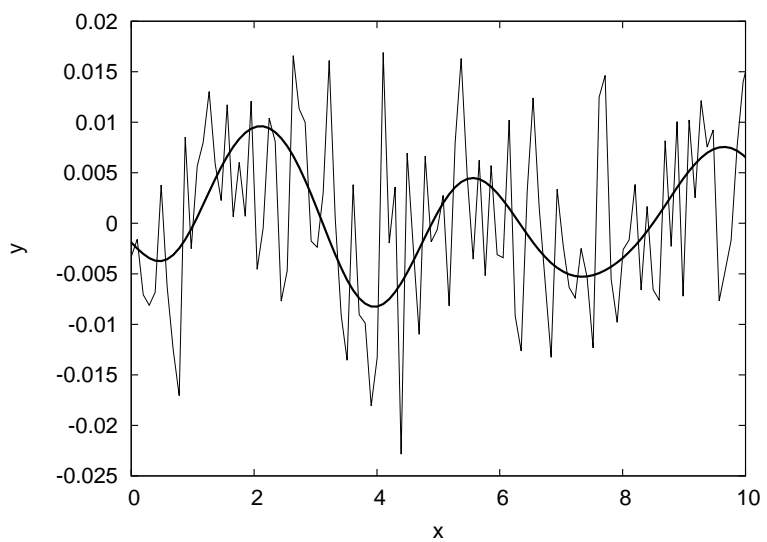

Figure 2: Initial bottom profile (thin line) and bottom profile after two oscillating cycles (thick line) $\left(R_{\delta}=33.87, s=1.27, R_{p}=2.99, \psi_{d}=12.96\right)$.
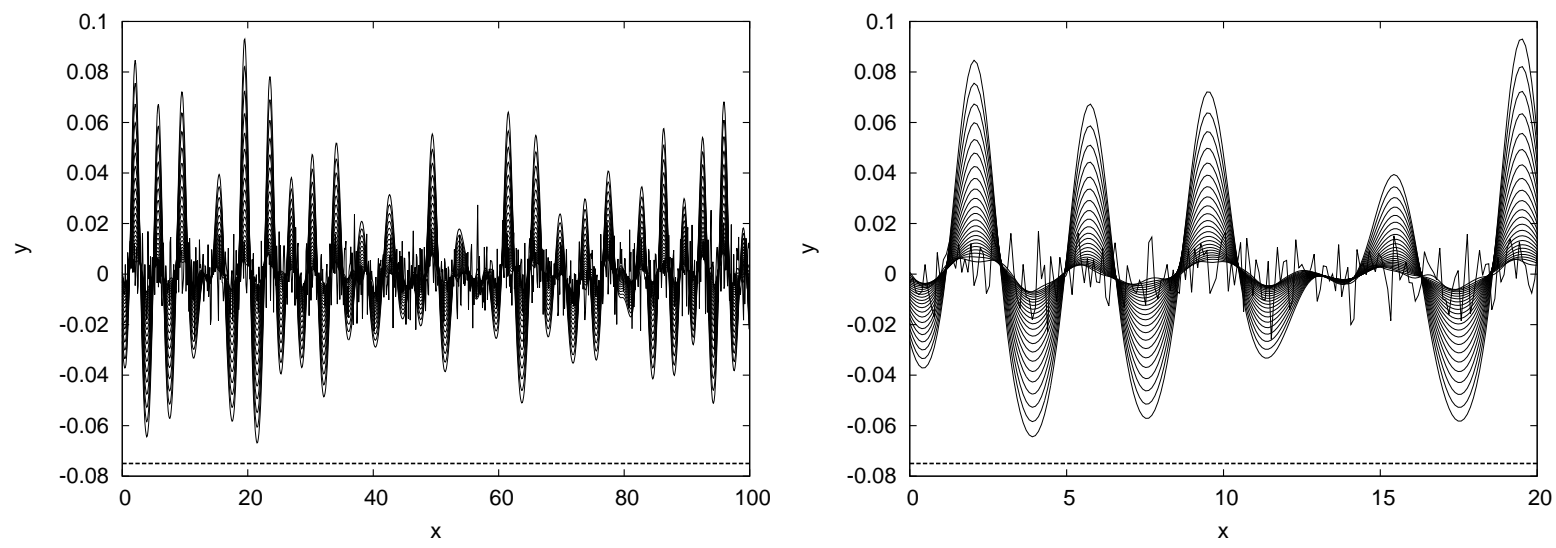

Figure 3: Initial time development of the bottom profile. The bottom profile is plotted every $\Delta \tau=0.468$, starting from $\tau=0$ until $\tau=11.22$, which corresponds to about 23 oscillating cycles $\left(R_{\delta}=33.87, s=1.27, R_{p}=2.99\right.$, $\left.\psi_{d}=12.96\right)$. The right hand panel is an enlargement of the region $0 \leq x \leq$ 20 . 


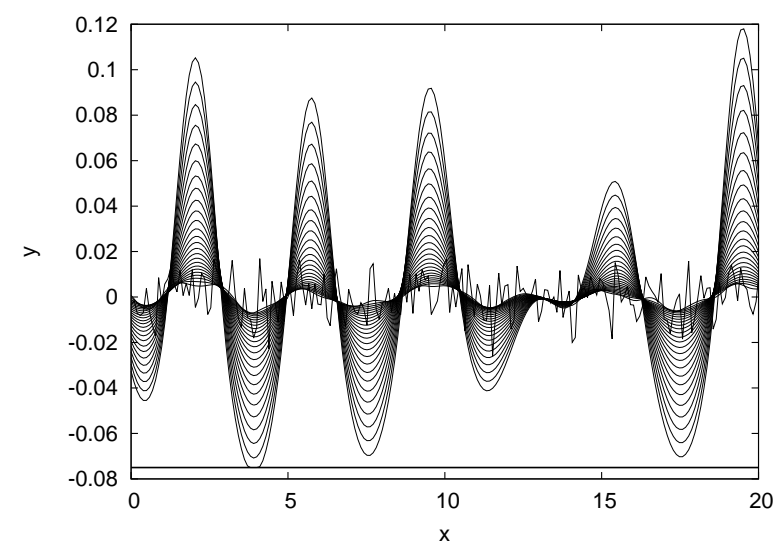

Figure 4: Time development of the bottom profile. The bottom profile is plotted every $\Delta \tau=0.468$, starting from $\tau=0$ until $\tau=12.16$, which corresponds to about 25 oscillating cycles $\left(R_{\delta}=33.87, s=1.27, R_{p}=2.99\right.$, $\left.\psi_{d}=12.96\right)$.

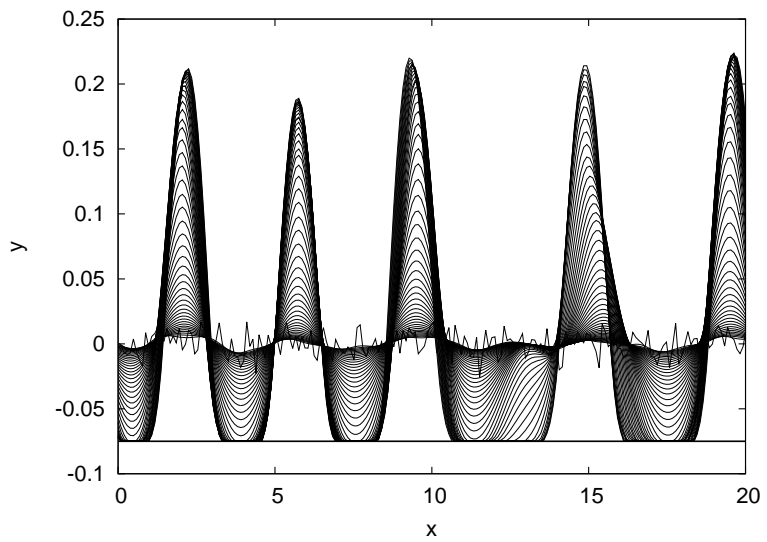

Figure 5: Time development of the bottom profile. The bottom profile is plotted every $\Delta \tau=0.468$, starting from $\tau=0$ until $\tau=22.91$, which corresponds to about 47 oscillating cycles $\left(R_{\delta}=33.87, s=1.27, R_{p}=2.99\right.$, $\left.\psi_{d}=12.96\right)$. 

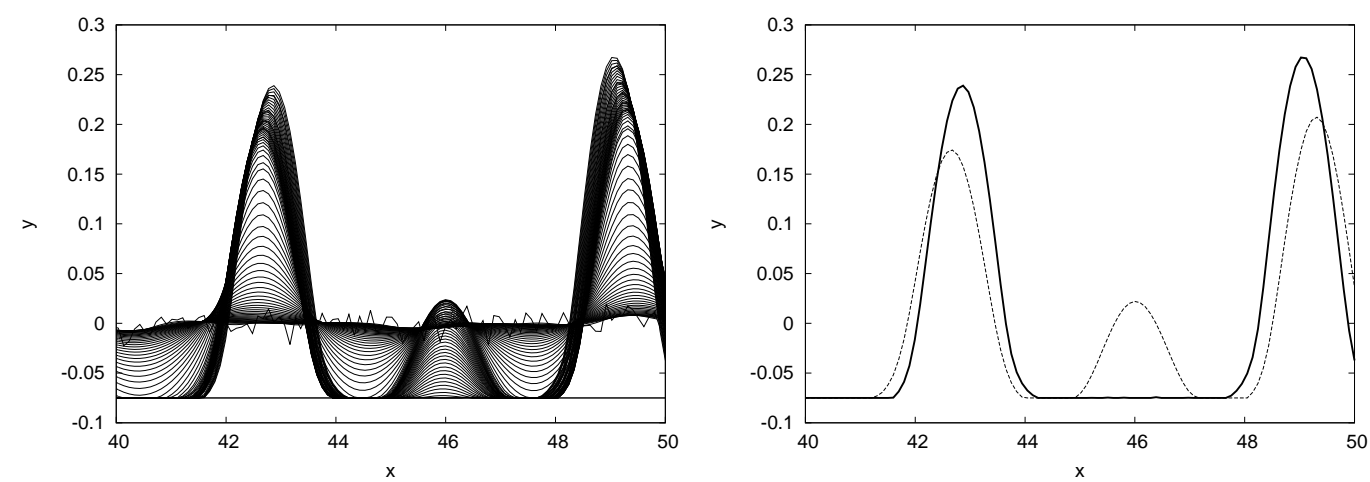

Figure 6: Details of the time development of the bottom profile for $40 \leq x \leq$ 50. In the left hand panel the bottom profile is plotted every $\Delta \tau=0.468$, starting from $\tau=0$ until $\tau=34.61$, which corresponds to about 70 oscillating cycles $\left(R_{\delta}=33.87, s=1.27, R_{p}=2.99, \psi_{d}=12.96\right)$. In the right hand panel the broken line is the bottom profile after about 2.5 cycles and the thick continuous line is the profile after about 70 cycles.
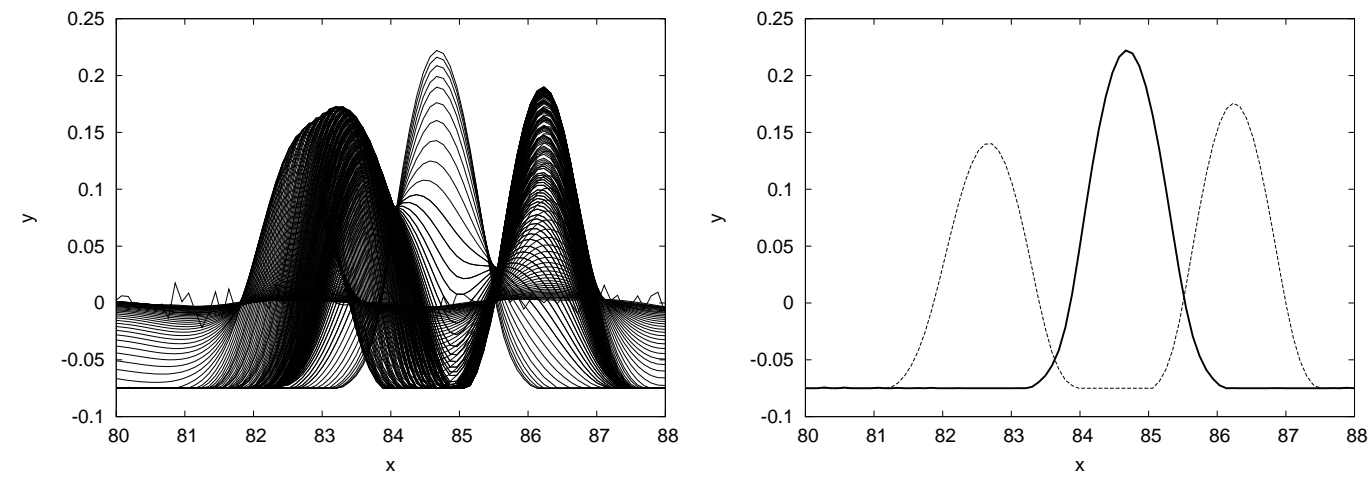

Figure 7: Details of the time development of the bottom profile for $80 \leq$ $x \leq 90$. In the left hand panel the bottom profile is plotted every $\Delta \tau=$ 0.468 , starting from $\tau=0$ until $\tau=72.95$, which corresponds to about 148 oscillating cycles $\left(R_{\delta}=33.87, s=1.27, R_{p}=2.99, \psi_{d}=12.96\right)$. In the right hand panel the broken line is the bottom profile after about 112 cycles and the thick continuous line is the profile after about 148 cycles. 


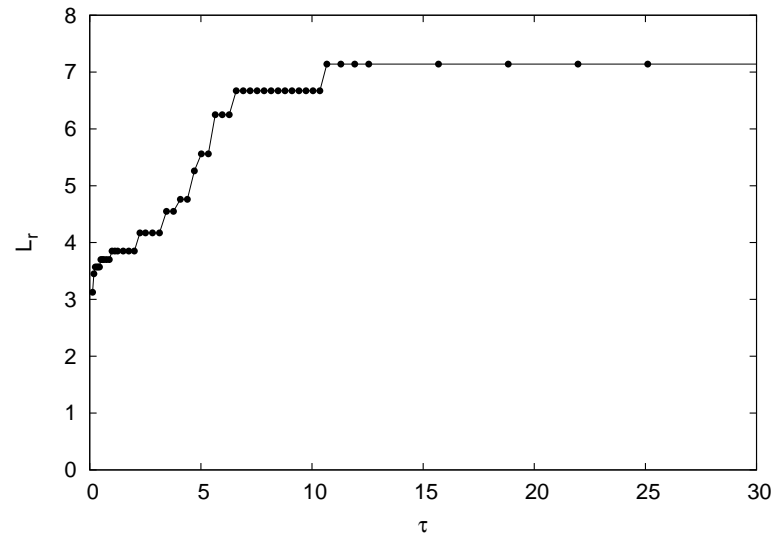

Figure 8: Dimensionless (spatially averaged) crest-to-crest distance of the bottom forms provided by the model as function of the number of fluid oscillations $\left(R_{\delta}=33.87, s=1.27, R_{p}=2.99, \psi_{d}=12.96, \Delta=0.075\right)$. 

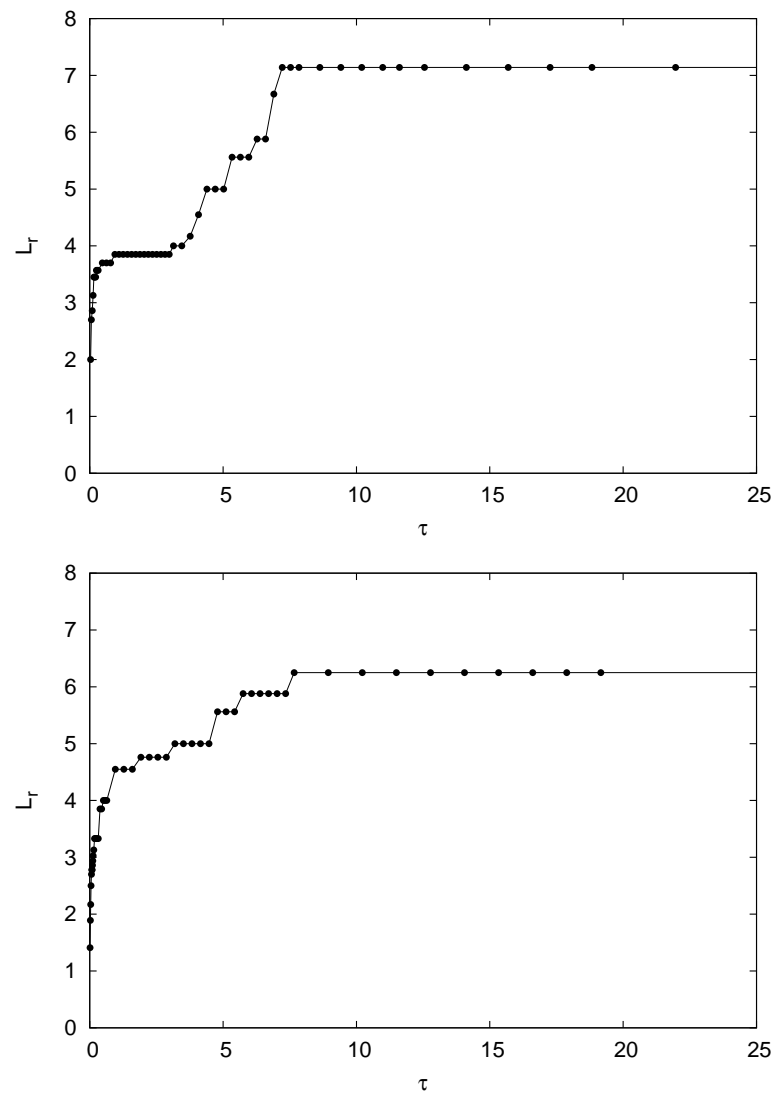

Figure 9: Dimensionless (spatially averaged) crest-to-crest distance of the bottom forms provided by the model as function of the number of fluid oscillations. Top panel: $R_{\delta}=33.87, s=1.27, R_{p}=2.99, \psi_{d}=12.96$, $\Delta=0.1\left(h^{*}=10 \mathrm{~cm}\right)$; Bottom panel: $R_{\delta}=25.30, s=1.27, R_{p}=2.99$, $\psi_{d}=7.84, \Delta=0.1\left(h^{*}=12.5 \mathrm{~cm}\right)$. 


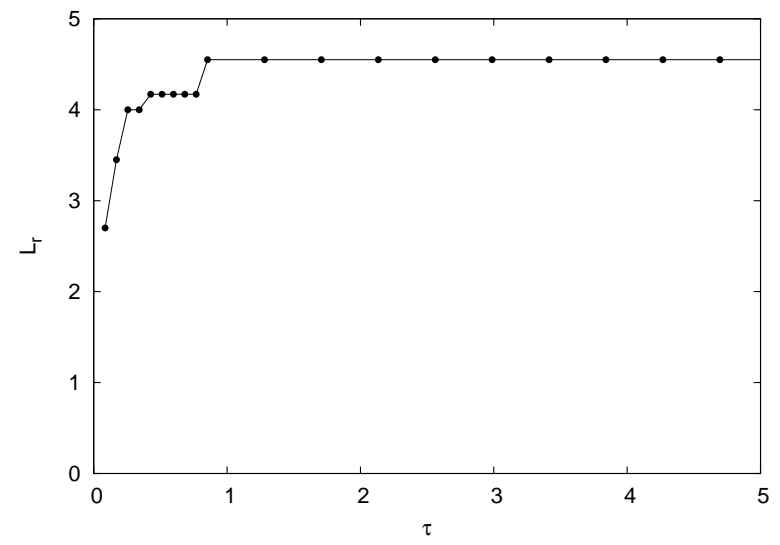

Figure 10: Dimensionless (spatially averaged) crest-to-crest distance of the bottom forms provided by the model as function of the number of fluid oscillations $\left(R_{\delta}=46.64, s=1.27, R_{p}=2.99, \psi_{d}=22.47, h^{*}=7.5, \Delta=0.1\right)$.

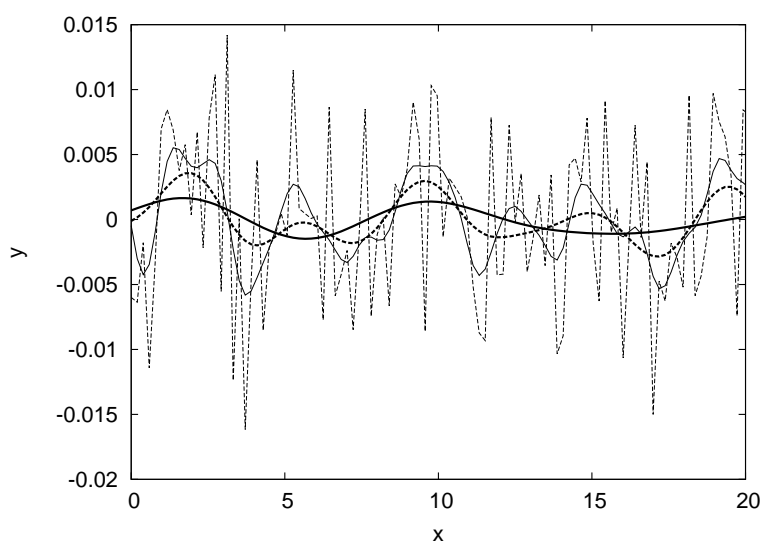

Figure 11: Bottom profile at $\tau=0$ (broken thin line), $\tau=0.016$ (continuous thin line), $\tau=0.16$ (broken thick line), $\tau=1.60$ (continuous thick line) $\left(R_{\delta}=16.94, s=1.27, R_{p}=2.99, \psi=3.23\right)$. 


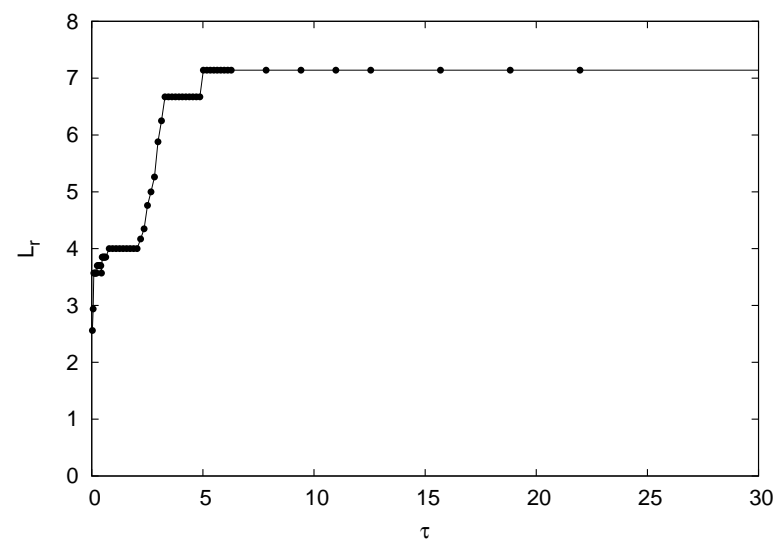

Figure 12: Dimensionless (spatially averaged) crest-to-crest distance of the bottom forms provided by the model as function of the number of fluid oscillations $\left(R_{\delta}=33.87, s=1.27, R_{p}=2.99, \psi_{d}=12.96, \Delta=0.15\right)$.

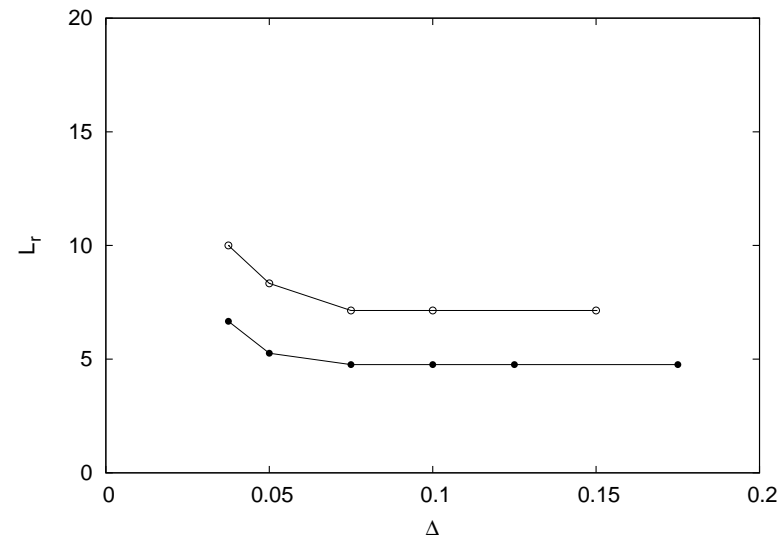

Figure 13: Dimensionless (spatially averaged) crest-to-crest distance at equillibrium as function of the thickness $\Delta$ of the sediment layer. White points $R_{\delta}=33.87, s=1.27, R_{p}=2.99, \psi_{d}=12.96$; black points $R_{\delta}=50.81, s=$ $1.27, R_{p}=2.99, \psi_{d}=29.05$. 


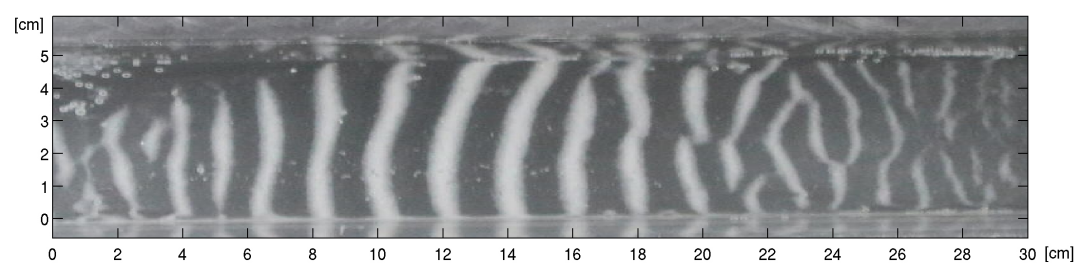

Figure 14: Example of the morphological patterns observed during the experiments (top view). The sediments appear as white regions while the rigid bottom appears black. At the top and at the bottom of the photo, the lateral walls reflect the bottom forms. The crest-to-crest distance can be readily estimated by taking into account that the lateral walls are $5 \mathrm{~cm}$ apart. To help the reader to estimate the size of the bedforms, horizontal and vertical axes are added to the photo but the numerical values they indicate are approximated because of the distortion of the camera lens.
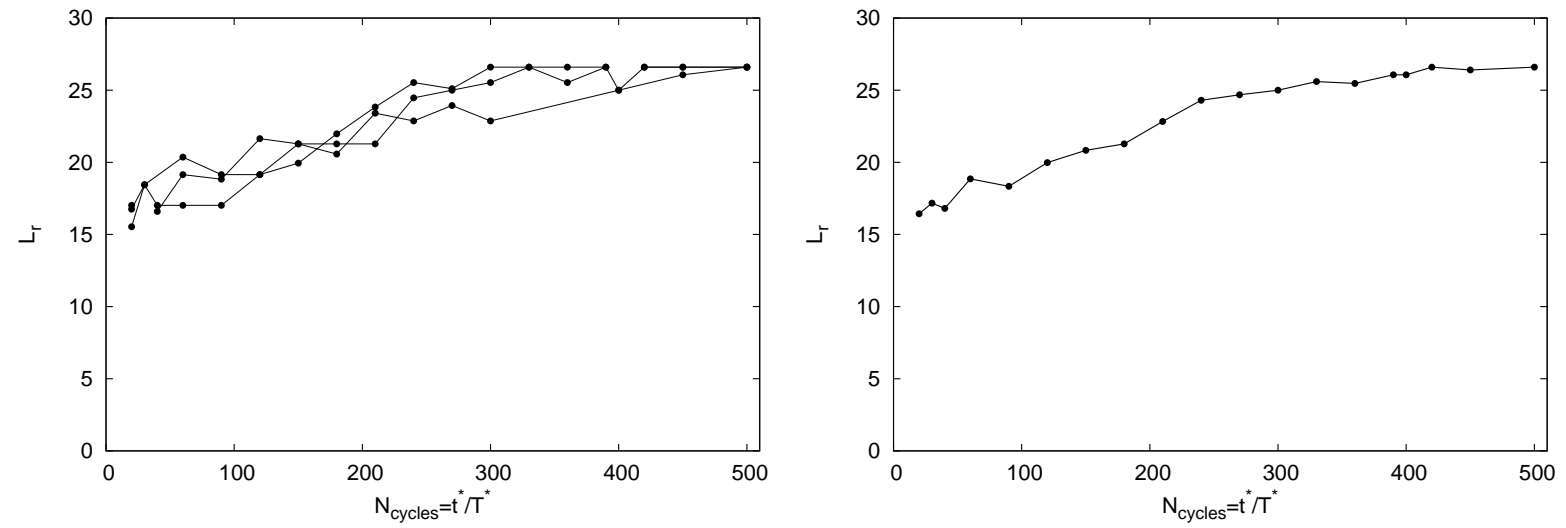

Figure 15: Dimensionless (spatially averaged) crest-to-crest distance plotted versus the number of fluid oscillations for $h^{*}=10 \mathrm{~cm}, H^{*}=2 \mathrm{~cm}, V^{*}=0.5$ $\mathrm{cm}^{3}\left(R_{\delta}=33.87, s=1.27, R_{p}=2.99, \psi_{d}=12.93\right)$. Left hand panel: results of three different experiments. Right hand panel: ensemble average value. 

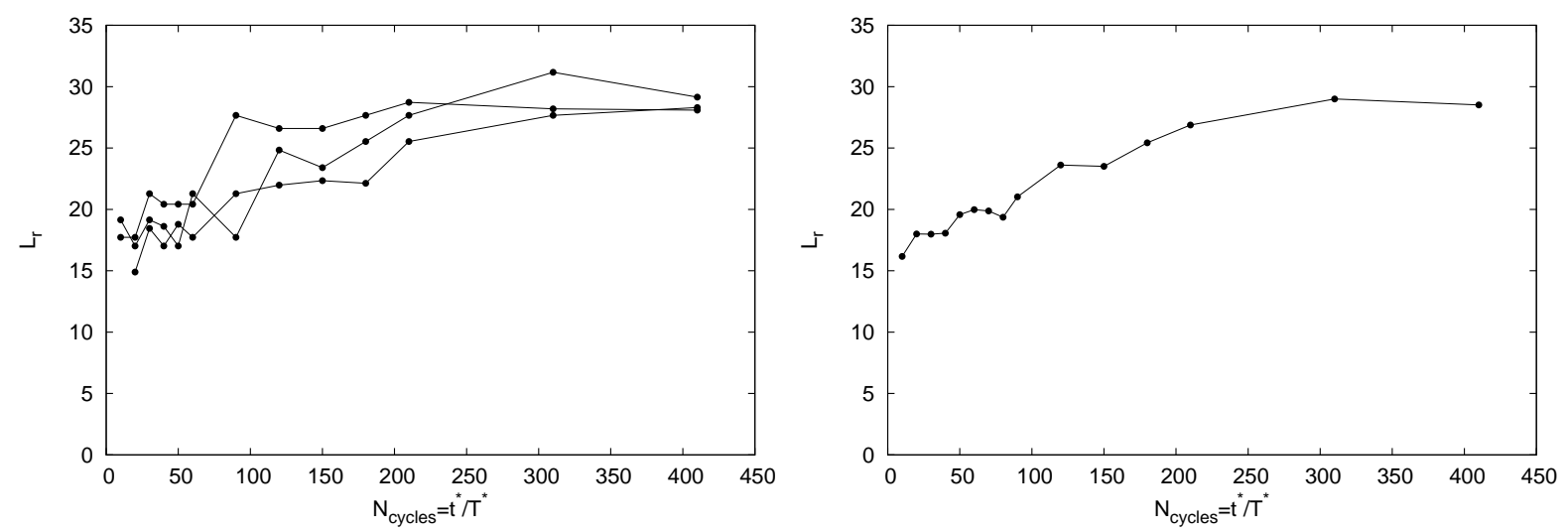

Figure 16: Dimensionless (spatially averaged) crest-to-crest distance plotted versus the number of fluid oscillations for $h^{*}=10 \mathrm{~cm}, H^{*}=2 \mathrm{~cm}, V^{*}=1$ $\mathrm{cm}^{3}\left(R_{\delta}=33.87, s=1.27, R_{p}=2.99, \psi_{d}=12.93\right)$. Left hand panel: results of three different experiments. Right hand panel: ensemble average value.
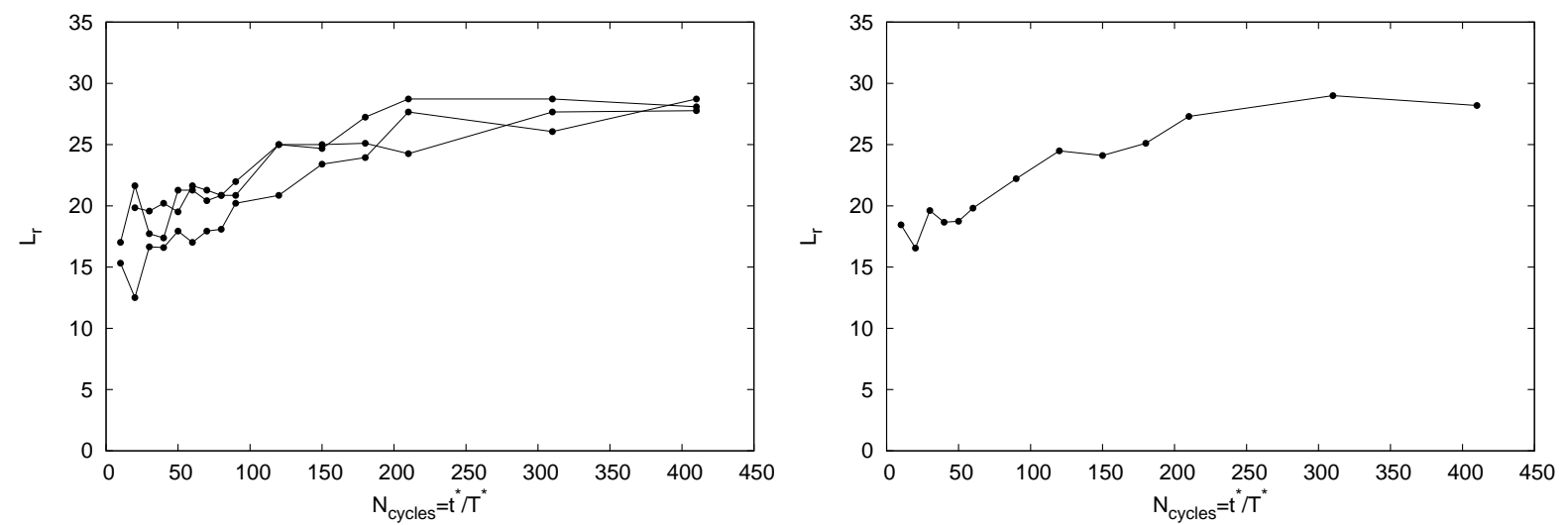

Figure 17: Dimensionless (spatially averaged) crest-to-crest distance plotted versus the number of fluid oscillations for $h^{*}=10 \mathrm{~cm}, H^{*}=2 \mathrm{~cm}, V^{*}=2$ $\mathrm{cm}^{3}\left(R_{\delta}=33.87, s=1.27, R_{p}=2.99, \psi_{d}=12.93\right)$. Left hand panel: results of three different experiments. Right hand panel: ensemble average value. 


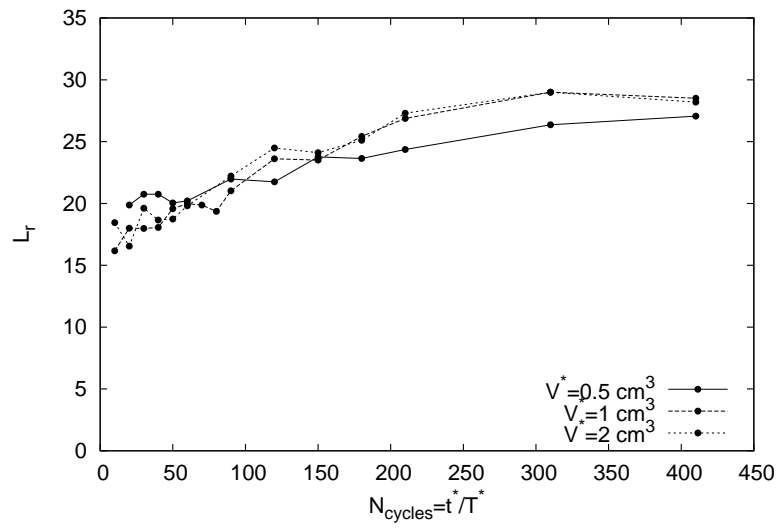

Figure 18: Ensenble averaged value of the dimensionless (spatially averaged) crest-to-crest distance plotted versus the number of fluid oscillations for $h^{*}=$ $10 \mathrm{~cm}, H^{*}=2 \mathrm{~cm}\left(R_{\delta}=33.87, s=1.27, R_{p}=2.99, \psi_{d}=12.93\right.$ and different volumes of sediments spread on the bottom, namely $V^{*}=0.5 \mathrm{~cm}^{3}, V^{*}=1$ $\mathrm{cm}^{3}$, and $V^{*}=2 \mathrm{~cm}^{3}$
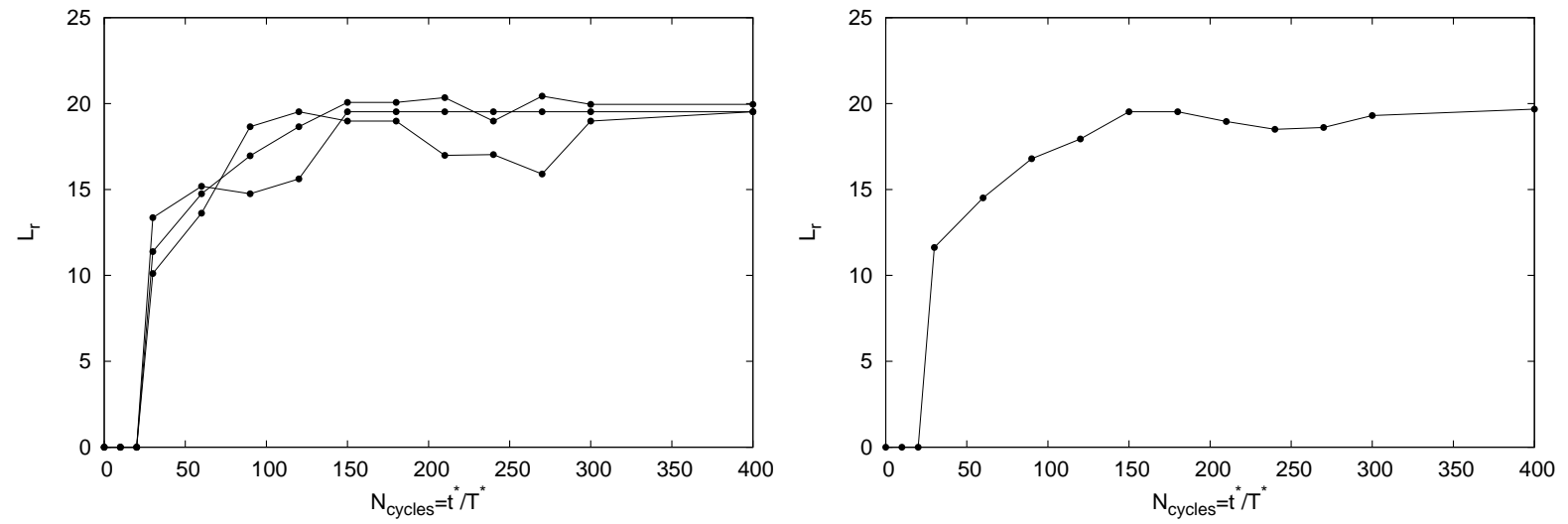

Figure 19: Dimensionless (spatially averaged) crest-to-crest distance plotted versus the number of fluid oscillations for $h^{*}=12.5 \mathrm{~cm}, H^{*}=2 \mathrm{~cm}, V^{*}=1$ $\mathrm{cm}^{3}\left(R_{\delta}=25.29, s=1.27, R_{p}=2.99, \psi_{d}=7.59\right)$. Left hand panel: results of three different experiments. Right hand panel: ensemble average value. 

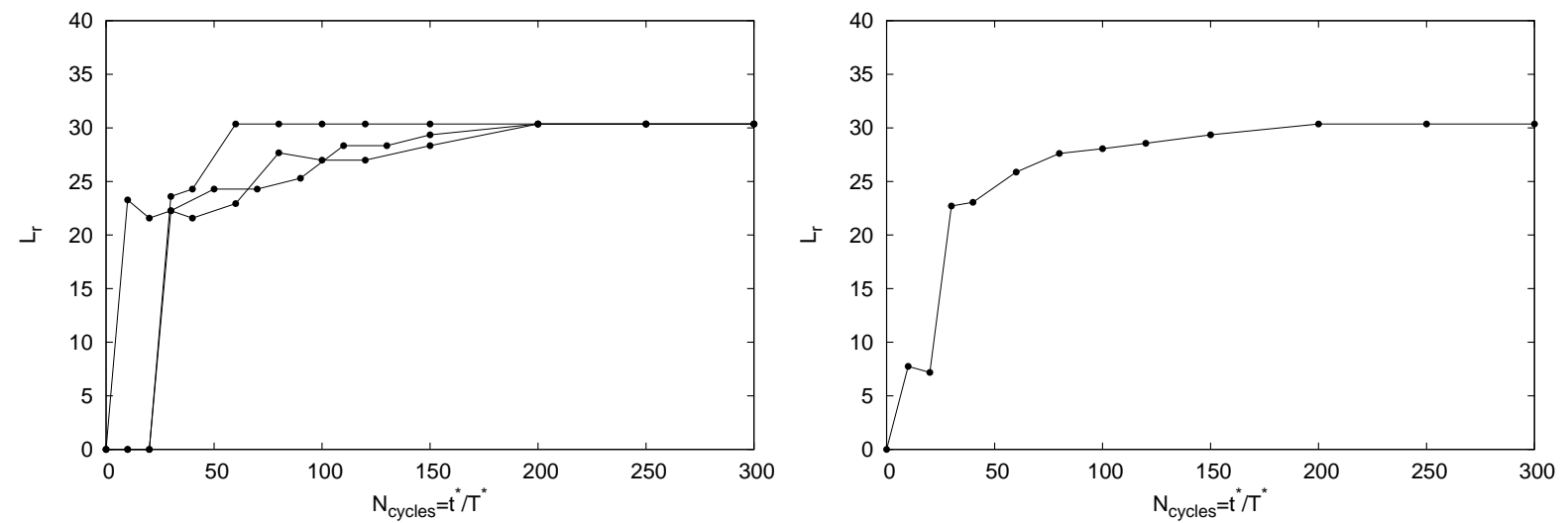

Figure 20: Dimensionless (spatially averaged) crest-to-crest distance plotted versus the number of fluid oscillations for $h^{*}=7.5 \mathrm{~cm}, H^{*}=2 \mathrm{~cm}, V^{*}=1$ $\mathrm{cm}^{3}\left(R_{\delta}=46.64, s=1.27, R_{p}=2.99, \psi_{d}=22.47\right)$. Left hand panel: results of three different experiments. Right hand panel: ensemble average value. 